\title{
The Variation of Action Potential and Impedance in Human Skeletal Muscle during Voluntary Contraction
}

\author{
Ten-Jen Liao and Hiroyasu Nishikawa* \\ Department of Oriental Medicine and ${ }^{*}$ Department of \\ Physiology, Meiji College of Oriental Medicine, Kyoto 629- \\ 03
}

\begin{abstract}
Liao, T.-J. and Nishikawa, H. The Variation of Action Potential and Impedance in Human Skeletal Muscle during Voluntary Contraction. Tohoku J. Exp. Med., 1994, 173 (3), 303-309 — We used an insulated acupuncture needle to deliver a $20 \mathrm{kHz}$ alternative current to detect the changes on the electromyogram (EMG) and deep muscle impedance. We found that impedance increased (with a duration of $224.1 \pm 75.3 \mathrm{msec}$ ) and then decreased (with a duration of $293.4 \pm 97.3$ $\mathrm{msec}$ ) after motor unit action potential (MUAP) firing during isometric voluntary contraction (VC) of the tibialis anterior muscle (TAM). We divided the positive peak into pattern I (with a latency range of $\geqq 30 \mathrm{msec}$ and $\leqq 75 \mathrm{msec}$ ) and pattern II (with a latency range of $>75 \mathrm{msec}$ and $\leqq 140 \mathrm{msec}$ ). These patterns of change corresponded to the contraction times of tension caused by the electrical stimulation and VC. These impedance changes may be caused by a change in the extracellular and intracellular fractions, and may reflect the variance of tension intensity in the muscle tissue. — voluntary contraction; action potential; impedance; insulated acupuncture needle
\end{abstract}

Impedance changes in muscle during contraction have been studied by Dubussion (1936), who reported that not strictly isometric contraction of a muscle is accompanied by a lowering of impedance, which is proportional to the shortening of fibers. Bozler (1935) also reported that the impedance increased on contraction; it also increased in iodoacetic acid rigor, and decreased in chloroform rigor. These findings suggested that the impedance change varies with the conditions (mechanical shortening or chemical process) in the living muscle. For two decades, the impedance has been cotinuously recorded and directly used for the calculation of the extracellular space (Pelligrino et al. 1981; Korf and Postema 1988). Although these impedance changes have been described, the relationship between muscle activity and impedance change is still unknown. Herein, we examined the relationship between the muscle activity and impedance change of the tibialis anterior muscle (TAM) during voluntary contraction (VC).

Received January 13, 1994; revision accepted for publication June 1, 1994.

Address for reprents: Dr. T.J. Liao, Department of Oriental Medicine, Meiji College of

Oriental Medicine, Kyoto 629-03, Japan. 
We conclude that the impedance increases and then decreases after motor unit active potential (MUAP) firing immediately, and according to the change of latencies we divided these variances into two patterns. Each pattern may be dominated by a different muscle fiber with a different contraction time, respectively. Therefore, by recording muscle impedance changes we determined the type of muscle fiber and the rough variation of the muscle tension.

\section{Materials and Methods}

Studies were conducted on 12 adult volunteers (male, mean 20 years) who were neurologically normal. They sat in a comfortable chair and put their foot on floor naturally in an electrically shielded room, and adjusted their posture so that no muscle activity was observed in the EMG monitored on an oscilloscope.

The recording electrode which is a sterile insulated acupuncture needle (200 $\mu \mathrm{m} \phi$, coated with formvar exposed tip about $1 \mathrm{~mm}$ ), was inserted into the right Tusanli acupoint (S 36, one finger breadth lateral to the lower border of the tuberositas tibiae, about $9 \mathrm{~cm}$ below the knee joint) (Stux and Pomeranz 1987). After needle insertion, the needle was manipulated until the fastest rise-times were secured in the main spikes of the lowest threshold MUAP (Warmolts and Engel 1972) during VC. When starting the experiment, the subjects maintained balances of their heels on the floor and then lifted up the dorsal foot from the floor to less than 5 degrees as the lowest VC, 5 to 10 degrees as a mild VC, and more than 10 degrees as a vigorous VC. The lowest VC was performed to produce MUAP firing, and as soon as the subject heard the burst sound (monitor by an oscilloscope, VC11; Nihon Kohden, Tokyo, and a speaker) VC was stopped. VC was performed for $10 \mathrm{~min}$ (per $10 \mathrm{sec}$ a time) of each subject.

In the recording procedure, we measured TAM impedance, EMG and zeta potential changes simultaneously during VC. For these measurements we delivered an alternating current of $100 \mathrm{nA}$ at a frequency of $20 \mathrm{kHz}$ by a locally

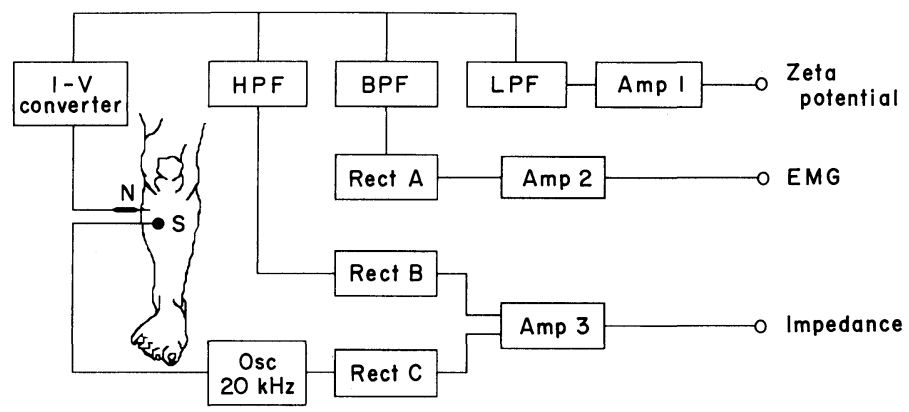

Fig. 1. Block diagram of the zeta potential, EMG and impedance measurments. I-V converter, current-voltage converter; HPF, high-pass filter; BPF, bandpass filter; LPF, low-pass filter; Rect, full wave rectifier; Amp, amplifier; Osc, oscillator (supplied $20 \mathrm{kHz}$ sine wave); $\mathrm{N}$, insulated acupuncture needle (as a leading electrode); S, surface electrode (as a reference). 
made device; this current does not cause any activation of the muscle (Fig. 1) (Liao 1992). In this device, a $20 \mathrm{kHz}$ carrier was put on the surface of TAM by the surface electrode as a reference. These three signals of $20 \mathrm{kHz}$ carrier, EMG and needle moving (named as zeta potential) will pass the tip of the insulated needle electrode and input to a current-voltage converter during $\mathrm{VC}$, then a low-pass filter $(10 \mathrm{~Hz})$, a band-pass filter $(10 \mathrm{~Hz}$ to $5 \mathrm{kHz})$ and a high-pass filter $(5 \mathrm{kHz})$ were used to separate the zeta potential and the EMG from the $20 \mathrm{kHz}$ carrier, and then to be amplified or rectified (full wave) then to be amplified and recorded as zeta potential, EMG and impedance. The power spectrum range of the EMG was approximately between several $\mathrm{Hz}$ and $3 \mathrm{kHz}$, with the most desirable frequency range for recording being 20 to $200 \mathrm{~Hz}$ (John 1979), and the zeta potential (Goton 1957) of the electrode movement was less than $2 \mathrm{~Hz}$ in this study. In this experiment, MUAP activated from the lowest VC up through mild $\mathrm{VC}$ or vigorous $\mathrm{VC}$ were observed, All of these signals were recorded by a data recorder (XR-50; TEAC, Tokyo), and only one single discharge was analyzed by VC-11 with a QP-110J program (Nihon Kohden).

\section{RESUlts}

In the above experiments, MUAP was activated from the minimal to the mild or vigorous effort which caused impedance changes. Usually, the impedance changes are described as an upward (increase) and then a downward (decrease) flection with a certain latency after firing MUAP (Fig. 2), and these peak latencies and peak amplitudes were different in each volunteer. The maximum procedure of this impedance change ended within $1 \mathrm{sec}$, and the duration of the positive waves was always shorter than the negative one. A steady rhythmic low frequency (range from 6 to 10/sec) MUAP was acitivated on minimal to mild VC (Fig. 3a-c). In the period of VC, the zeta potential was always recorded and changed only of the initial point and end of the VC. The impedance appeared with a delay time after EMG firing. Different positive peak values of impedance were plotted and showed that many of the components were distributed in the

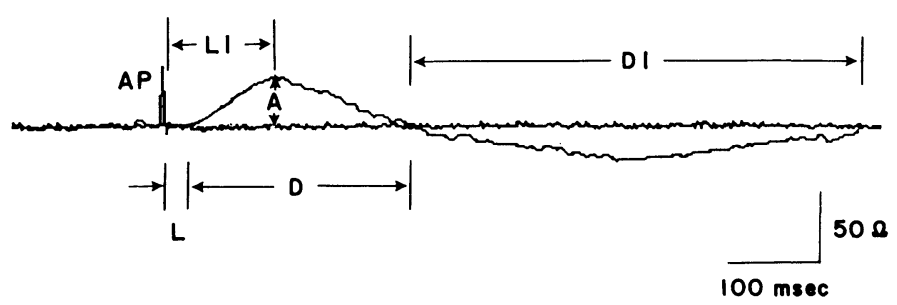

Fig. 2. Relationship between action potential and the muscle impedance recorded simultaneously from TAM during VC.

$\mathrm{AP}$, action potentional; A, amplitude of positive wave; $\mathrm{D}$, duration of positive wave; $\mathrm{D} 1$, duration of negative wave; $\mathrm{L}$, delay time; L1, positive peak latency. 


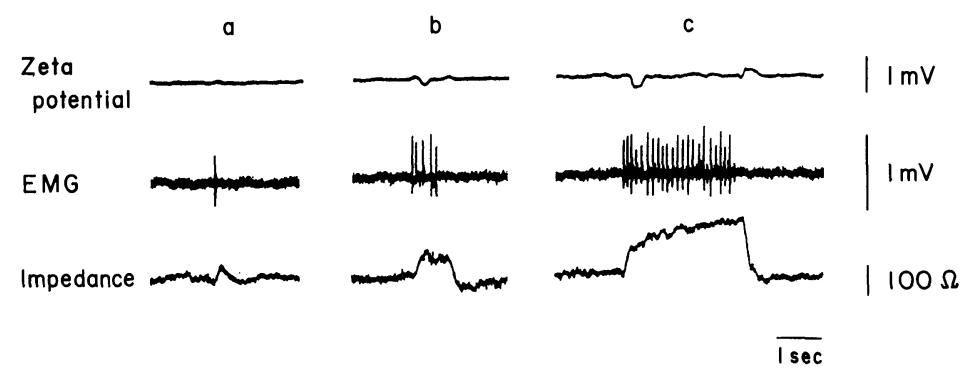

Fig. 3. The zeta potentials (upper traces), EMGs (middle traces) and impedances change (bottom traces) recorded simultaneously from TAM during the lowest $\mathrm{VC}$ (a) and mild VC (b, c).

The former shows a single and the latter shows two rhythmic low frequency MUAP.

TABLE 1. The data component of impedance recorded from TAM during VC

\begin{tabular}{lcc}
\hline \multicolumn{1}{c}{ Data item } & mean \pm s.D. & $n$ \\
\hline Delay time (msec) & $8.0 \pm 6.0$ & 130 \\
Positive peak amplitude $(\Omega)$ & $110.5 \pm 94.0$ & 130 \\
Positive peak latency $(\mathrm{msec})$ & $66.3 \pm 21.3$ & 130 \\
Positive wave duration $(\mathrm{msec})$ & $224.8 \pm 75.3$ & 130 \\
Nagative wave duration $(\mathrm{msec})$ & $293.4 \pm 97.3$ & 123 \\
\hline
\end{tabular}

The values were mean and S.D. of delay time after MUAP firing, positive peak amplitude, peak latency, wave duration, and negative wave duration recorded from TAM during VC.

short latencies area (Fig. 4). Most of the impedances changed from the range of $30 \Omega$ to $300 \Omega$ during VC. Table 1 shows the average value and standard deviation of positive peak amplitude, peak latency, wave duration, delay time after MUAP firing and negative wave durations. Upward impedance increased with a delay time of $8.0 \pm 6.0 \mathrm{msec}$ after MUAP firing, and the average duration of negative waves was longer than that of the positive waves. Fig. 5 shows the distribution of latency percentage content (from Fig. 4). Short latencies (with a latency range of $\geqq 30 \mathrm{msec}$ and $\leqq 75 \mathrm{msec}$ ) showed a high percentage content, and long latencies (with a latency range of $>75 \mathrm{msec}$ and $\leqq 140 \mathrm{msec}$ ) showed a low percentage content.

\section{Discussion}

The size principle (Henneman 1957) is applied to both slow and rapid VCs in man (Desmedt and Godaux 1977), and according to this principle type 1 muscle fibers (red muscle) are fired under mild VC and type 2 (white muscle) are fired under vigorous or sudden VC (Warmolts and Engel 1972). In the case of mild VC in this study, the impedance duration was longer than the firing interval that 


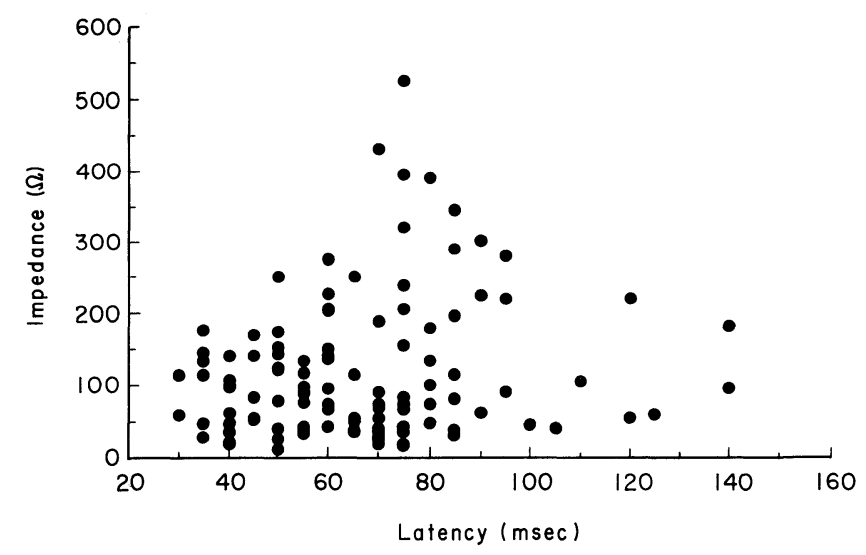

Fig. 4. The peak amplitude distribution of each positive latency of impedance recorded from TAM during $\mathrm{VC}(n=130)$.

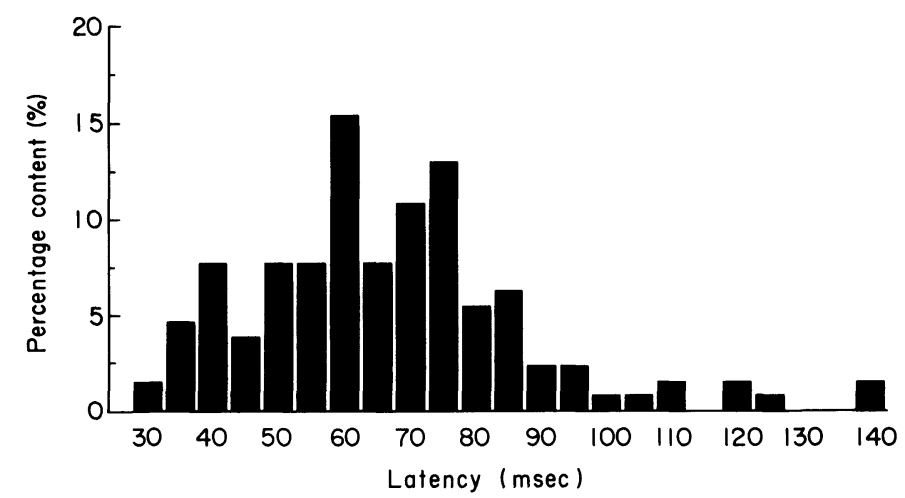

Fig. 5. The percentage content of each positive peak's latency of impedance recorded from TAM during VC.

produced the impedances fusing together (Fig. $3 \mathrm{~b}$ and $3 \mathrm{c}$ ). These MUAP and impedance change patterns resembled the relationship between MUAP and contraction time (Buchthal and Schmalbruch 1970; Majima 1978). The electrical impedance of cells and tissues is largely determined by the properties of the surface membrane and these properities in turn depend on the activity of the cell as a whole (Bozler 1935). In this study we just measured the relative impedance change, before, during and after VC. Because any pressure put on the muscle or change of muscle shape will cause an impedance change (Liao 1992), we offset the direct current level and always kept the same impedance level before and after VC.

The simplest explanation for the increase of impedance on contraction would therefore be that it is an increase of the volume concentration of the cells due to swelling (Bozler 1935). When mechanical shortening is completely avoided, the 
impedance changes are effected by the chemical processes known to occur in the muscle (Dubussion 1936). In this study, whether these impedance changes are effected by a chemical process or not during VC remains unknown. However, because the evidence to support this suggestion is lacking, the zeta potential change and MUAP firing are always observed at the same time in the process of VC. These zeta potential changes indicate that the tip of the needle electrode moved in the muscle during $\mathrm{VC}$, for this moving will cause zeta potential production (Goton 1957). These findings suggest that the mechanical shortening causes the impedance change.

Most of the individuals in this study showed an impedance increase then decrease, and a few showed an impedance decrease then increase. This may be because the $\mathrm{VC}$ is not sufficiently strict to offer a perfect isometric condition. In strictly isometric conditions the contraction is accompanied by a rise in impedance (Dubussion 1936). Nevertheless, we found that the impedance change patterns (duration and amplitude) resembled each other whether there was increase then decrease or decrease then increase. This positive muscle impedance change may reflect the change of muscle tension at the same time, which is caused by a variance in the extracellular and intracellular fractions, because the mechanical shortening may cause cell volume concentration during VC. VC was performed within a short time (10 min) so that the cell swelling causing an impedance change would not need to be considered. The zeta potential change is always observed after the end of the VC. This may suggest that the muscle relaxes suddenly and causes a negative impedance change.

The different types of change of muscle impedance might be caused by the different muscle fibers that attend the contraction. TAM contains two different fibers; a fast-twitch-glycolytic fiber (FG fiber) and a slow-twitch-oxidative fiber (SO fiber) (Buchthal and Schmalbruch 1970; Peter et al 1972; Nagata 1984). The contraction time of SO fiber is longer than $60 \mathrm{msec}$, and that of the FG fiber is shorter than 60 msec (Buchthal and Schmalbruch 1970). In this study, we observed a short latency with a high percentage content $(76.9 \%, 100$ of 130 samples) of impedance changes in the range of $\geqq 30 \mathrm{msec}$ and $\leqq 75 \mathrm{msec}$, and a long latency with a low percentage content (23.1\%, 30 of 130 samples) of impedance changes in the range of $>75 \mathrm{msec}$ and $\leqq 140 \mathrm{msec}$ during VC (Fig 4). These values are in contradiction to the distribution rate of $73.4 \%$ and $26.6 \%$ for $\mathrm{FG}$ fibers and SO fibers of human TAM respectively (Nagata 1984). According to these findings we divided the impedance change components of $\geqq 30$ msec and $\leqq$ $75 \mathrm{msec}$ into pattern I, $>75 \mathrm{msec}$ and $\leqq 140 \mathrm{msec}$ into pattern II, and suggested that the FG fiber will activate the former and SO fiber activate the latter impedance change.

The difference in impedance latency and amplitude in each pattern may be caused by the different intensity of $\mathrm{VC}$ and the location of electrode, because movement of the electrode or slight increase in the stimulus caused a new fiber 
bundle to be activated (Buchthal and Schmalbruch 1970). The negative impedance change may reflect relaxation phase of the muscle change. By means of recording muscle impedance change we may determine the type of muscle fiber and the rough variation of the muscle tension.

\section{Acknowledgments}

We thank Mr. H. Hiraguri for his excellent technical assistance, and Mr. K. Mukaiyama, Mr. S. Mukaiyama, Mr. N. Hara, Mr. S. Hayashi, Mr. S.L. Wang and Mr. H. Nakanishi for their valuable help.

\section{References}

1) Bozler, E. (1935) The change of alternating current impedance of muscle of muscle produced by contraction. J. Cell Comp. Physiol., 6, 217-228.

2) Buchthal, F. \& Schmalbruch, H. (1970) Contraction times and fibre types in intact human muscle. Acta Physiol. Scand., 79, 435-452.

3) Desmedt, J. E. \& Godaux, E. (1977) Fast motor units are not preferential activated in rapid voluntary contractions in man. Nature, 267, 717-719.

4) Dubussion, M. (1936) Impedance change in muscle during contraction, and their possible relation to chemical processes. Arch. Int. Physiol., 42, 132-152.

5) Goton, M.Y. (1957) Junction potential. In: The Electrical Phenomenon and It's Application of Human, Nagaii Books Co., Tokyo, pp. 76-77.

6) Henneman, E. (1957) Relation between size of neurons and their susceptibility to discharge. Science, 126, 1345-1347.

7) John, R.B. (1979) Electromyogram, In: Physiological Basis of Medical Practice, the Williams and Wilkins Co., Baltimore, pp. 105-106.

8) Korf, J. \& Postema, F. (1988) Rapid shrinkage of rat striatal extracellular space after local kainate application and ischemia recorded by impedance. J. Neurosci. Res., 19, 504-510.

9) Liao, T.J. (1992) Quantitative measurement of the acupuncture sensation caused by the acupuncture stimulation. Ann. Appl. Inform. Sci., 17, 31-48.

10) Majima, H. (1978) The contraction oaf skeletal muscle. In: Physiology. Bunkoudou, Tokyo, pp. 47-72.

11) Nagata, A. (1984) Motor unit. In: The Science of Muscle and Musclar Strength, Fumaidou, Tokyo, pp. 55-72.

12) Pelligrino, D., Almquist L.-O. \& Siesjo, B.K. (1981) Effect of insulin-indueced hypoglycemia on intracellular $\mathrm{PH}$ impedance in the cerebral cortex of the rat. Brain Res., 221, 129-147

13) Peter, J.B., Barnard, R.J., Edgerton, V.R., Gillespie, C.A. \& Stempel, K.E. (1972) Metabolic profiles of three fiber types of skeletal muscle in guinea pigs and rabbits. Biochemistry, 11, 2627-2633.

14) Stux, G. \& Pomeranz, B. (1987) Systematic description of channels and points. In: Acupuncture, Springer-Verlag Berlin Heidelberg, New York, pp. 77-211.

15) Warmolts, JR. \& Engel, W.K. (1972) Open-biopsy electromyography. 1. Correction of motor unit behavior with histochemical muscle fiber type in human limb muscle. Arch. Neurol., 27, 512-517. 